\title{
Method and Implementation of Accuracy Measurement on Rotation
}

\section{Angle of Antenna-driven Component}

\author{
Jiaping Song ${ }^{1, a^{*}}$ and Yushu Bian ${ }^{2, b}$ \\ ${ }^{1}$ School of Mechanical Engineering and Automation, Beihang University, China \\ ${ }^{2}$ School of Mechanical Engineering and Automation, Beihang University, China \\ a517871631@qq.com, b448316653@qq.com
}

Key words: antenna-driven component; accuracy measurement; photoelectric autocollimator; MFC Abstract: The antenna-driven component is an important unit used to drive an antenna to its prescribed position. In this paper, an accuracy method for measuring rotation angle of the antenna-driven component is proposed and the corresponding measurement equipment is developed. The working principles of accuracy measurement and error analysis are presented at detail. The PC application software associating with the measurement equipment is developed. The related experiments are conducted. The experiment results demonstrate the correctness and effectiveness of the method.

\section{Introduction}

The antenna-driven component is an important unit used to drive an antenna to its prescribed position. It is necessary to measure the rotation angle in the stage of product acceptance, product loading, and performance test during the development of driven component [1].

Usually, small angle measurement methods contain three categories, i.e. mechanical methods, electromagnetic methods and photoelectric methods [2-4]. Among these methods, most of mechanical methods and electromagnetic methods have to be implemented manually, and can only obtain limited measurement accuracy. In contrast to above methods, photoelectric methods possess such characteristics as non-contact and high sensitivity, thus have attracted much attention.

In this paper, an accuracy method for measuring rotation angle of the antenna-driven component is proposed and the corresponding measurement equipment is developed. The working principles of accuracy measurement and error analysis are presented at detail. The PC application software associating with the measurement equipment is developed. The related experiments are conducted. The experiment results demonstrate the correctness and effectiveness of the method.

\section{Scheme of Accuracy Measurement System}

The accuracy measurement system is designed based on the scheme of the turntable and Photoelectric Autocollimator. It is mainly composed of test platform, motion control unit, optical measurement unit, monitoring and information processing unit. Test platform mainly includes the turntable, optical platform, operating floor. Motion control unit used to control the movement of the turntable and drive component mainly includes industrial control, the turntable servo system, driven component motion servo system. Optical measurement unit used in the optical measurement and data acquisition mainly includes Photoelectric Autocollimator, plane mirror and related accessories. Monitoring and information processing unit is mainly responsible for human-computer interaction, information processing, power control, etc. The scheme of accuracy measurement on rotation angle of antenna-driven component is shown in Figure 1. 


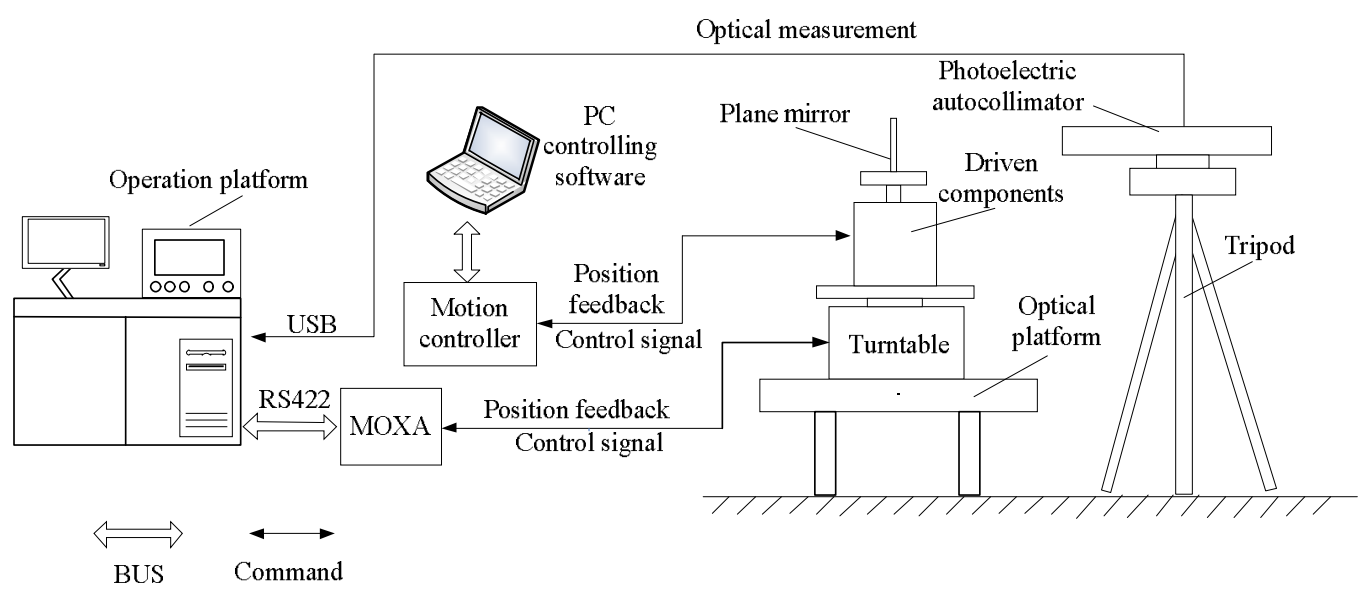

Fig.1 Scheme of accuracy measurement on rotation angle of antenna-driven component

\section{Principle of Accuracy Measurement System}

The following equations will be used to measure accuracy on rotation angle.

$$
X_{i}=P_{i}^{\prime}-P_{i} ; \quad \bar{X}=\frac{1}{m} \sum_{i=1}^{m} X_{i} ; \quad R=\sqrt{\frac{1}{m} \sum_{i=1}^{m} X_{i}^{2}} ; \quad \sigma=\sqrt{\frac{1}{m-1} \sum_{i=1}^{m}\left(X_{i}-\bar{X}\right)^{2}} .
$$

Where $X_{i}$ : position deviation; $\bar{X}$ : average of position deviation; $R$ : root mean square value of position deviation; $\sigma$ : standard deviation of position deviation; $P_{i}^{\prime}$ : actual position; $P_{i}$ : target position; $m$ : numbers of accuracy measurement; $i: 1,2, \ldots, m$.

The working principle of accuracy measurement scheme is addressed as follows. First, the antenna-driven component is instructed to rotate an angle. Obviously, the plane mirror will rotate the same angle as well. Second, the turntable is driven to rotate the same angle in the reverse direction. Third, the rotation angle of the turntable is measured using the autocollimator and the plane mirror. Finally, the error between the rotation angle of the antenna-driven component and that of the turntable is calculated. If motion accuracy of the turntable is high enough and can be viewed as "absolutely" accurate, then this error can be considered as the rotation angle accuracy of the antenna-driven component.

The initial value of Photoelectric Autocollimator on $\mathrm{X}$-axis is $\theta_{\mathrm{X}}$, while the initial angle of antenna-driven component is $\theta_{\mathrm{T}}$. The antenna-driven component is driven by controller to rotate an angle $\theta_{\mathrm{L}}$ using the software. The value of $\theta_{\mathrm{L}}$ is the initial angle of rotation of antenna-driven component. The angle of antenna-driven component $\theta_{\mathrm{T}}{ }^{\prime}$ will be recorded after finishing rotation. Then the turntable is driven to rotate the angle of $\theta_{\mathrm{L}}$ in the reverse direction by the commands of software. After all these finished, the value of Photoelectric Autocollimator on X-axis $\theta_{\mathrm{X}}{ }^{\prime}$ and the angle of turntable $\theta_{\mathrm{L}}{ }^{\prime}$ will be recorded. Assume that clockwise rotation is the positive direction. Then the following equations will be concluded.

$$
\begin{gathered}
\Delta c=\left(\theta_{\mathrm{X}}{ }^{\prime}-\theta_{\mathrm{X}}\right)-\left(\theta_{\mathrm{L}}{ }^{\prime}-\theta_{\mathrm{L}}\right) . \\
\Delta c_{b}=\left(\theta_{\mathrm{T}}{ }^{\prime}-\theta_{\mathrm{T}}-\theta_{\mathrm{L}}\right)-\left[\left(\theta_{\mathrm{X}}{ }^{\prime}-\theta_{\mathrm{X}}\right)-\left(\theta_{\mathrm{L}}{ }^{\prime}-\theta_{\mathrm{L}}\right)\right] .
\end{gathered}
$$

Where $\Delta c$ : angle deviation of antenna-driven component, $\Delta c_{b}$ : the encoder errors.

The following equations will also be concluded after test several times.

$$
\Delta c_{i}=\left(\theta_{\mathrm{X} i}^{\prime}-\theta_{\mathrm{X} i}\right)-\left(\theta_{\mathrm{L} i}^{\prime}-\theta_{\mathrm{L} i}\right)
$$




$$
\Delta c_{b i}=\left(\theta_{\mathrm{T} i}^{\prime}-\theta_{\mathrm{T} i}-\theta_{\mathrm{L} i}\right)-\left[\left(\theta_{\mathrm{X} i}^{\prime}-\theta_{\mathrm{X} i}\right)-\left(\theta_{\mathrm{L} i}^{\prime}-\theta_{\mathrm{L} i}\right)\right] .
$$

Where $\Delta c_{i}$ : angle deviation of antenna-driven component for the $i$ time, $\Delta c_{b i}$ : the encoder errors for the $i$ time; $i: 1,2, \ldots, m ; m:$ numbers of precision test.

Then the following equations will be concluded.

$$
R_{C}=\sqrt{\frac{1}{m}\left[\left(\theta_{\mathrm{X} i}^{\prime}-\theta_{\mathrm{X} i}\right)-\left(\theta_{\mathrm{L} i}^{\prime}-\theta_{\mathrm{L} i}\right)\right]^{2}} .
$$

Where $R_{C}$ : root mean square value of angle deviation of driven component after test $m$ times.

\section{Software Development and Implementation of Accuracy Measurement System}

The PC application software associating with the accuracy measurement system is developed by using Microsoft Visual Studio application, through which the functions of calibration of accuracy measurement system, angle measurement of antenna-driven component, data processing and storage, and the generation of test reports will be implemented. The main function modules on the PC application software of accuracy measurement system are shown in Figure 2. The process of accuracy measurement system is shown in Figure 3.

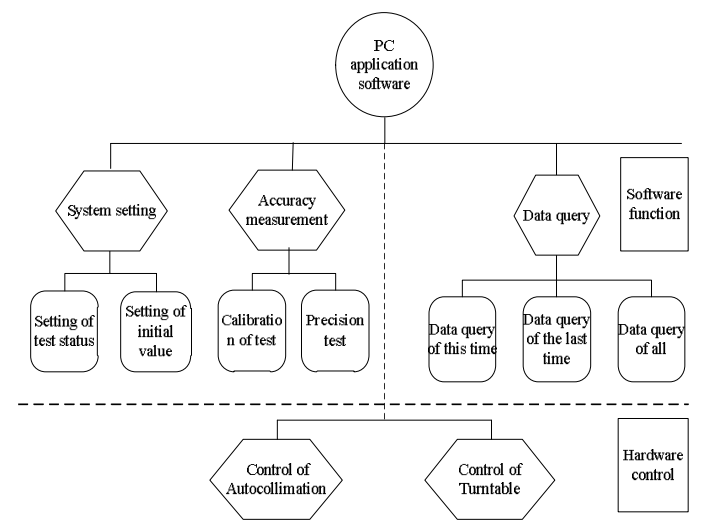

Fig.2 Main function modules on the PC software system

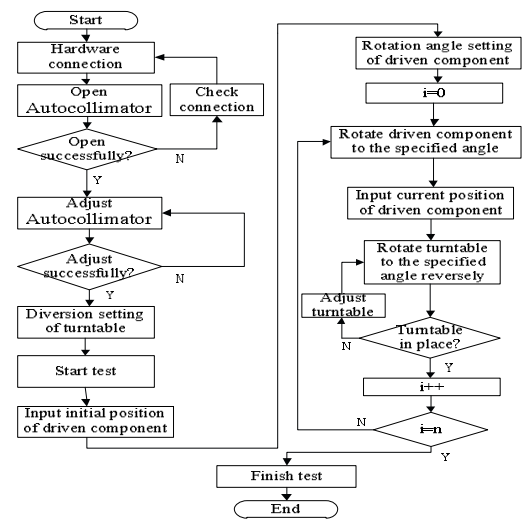

Fig.3 Process of accuracy measurement

\section{Result of Accuracy Measurement Experiment}

It is necessary to calibrate the accuracy measurement system before angle measuring of antenna-driven component. After finishing the angle measurement of driven component, the test report of experiment data will be generated to analyze and evaluate its performance. The experiment of accuracy measurement of driven component by using turntable, plane mirror and Autocollimator is shown in Figure 4. The result of accuracy measurement experiment is shown in Table1.

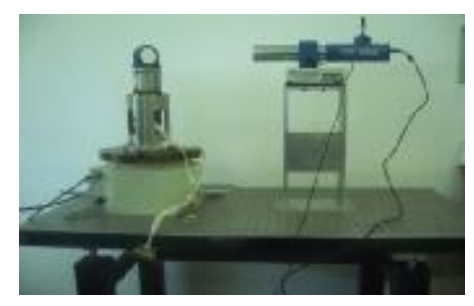

Fig.4 Experiment of accuracy measurement of antenna-driven component 
Table1 Result of accuracy measurement experiment

\begin{tabular}{|c|c|c|c|c|c|c|c|}
\hline NO. & $\begin{array}{c}\text { Rotation } \\
\text { angle of } \\
\text { turntable }\left(^{\circ}\right)\end{array}$ & $\begin{array}{l}\text { Position of } \\
\text { turntable }\left(^{\circ}\right)\end{array}$ & $\begin{array}{l}\text { Rotation angle } \\
\text { of driven } \\
\text { component }\left({ }^{\circ}\right)\end{array}$ & $\begin{array}{c}\text { Position of } \\
\text { driven } \\
\text { component }\left(^{\circ}\right)\end{array}$ & $\begin{array}{l}\text { Autocollimator } \\
\text { reading }\left({ }^{\prime \prime}\right)\end{array}$ & $\begin{array}{c}\text { Errors } \\
\text { of } \\
\text { encoder } \\
\quad\left({ }^{\prime \prime}\right)\end{array}$ & $\begin{array}{l}\text { position } \\
\text { deviation } \\
\text { (") }\end{array}$ \\
\hline 1 & -20.04950 & -20.04956 & 20.049504 & 53.83301 & -19.2 & 0.65 & -18.45 \\
\hline 2 & -20.04950 & -20.04958 & 20.049504 & 73.88855 & 26.2 & -23.09 & 27.02 \\
\hline 3 & -20.04950 & -20.04956 & 20.049504 & 93.93311 & -10.7 & -3.94 & -9.92 \\
\hline 4 & -20.04950 & -20.04956 & 20.049504 & 113.97766 & -31.6 & -0.89 & -30.81 \\
\hline 5 & -20.04950 & -20.04958 & 20.049504 & 134.02771 & -12.3 & -18.24 & -11.50 \\
\hline 6 & -20.04950 & -20.04958 & 20.049504 & 154.08325 & 17.7 & -26.57 & 18.56 \\
\hline 7 & -20.04950 & -20.04956 & 20.049504 & 174.11682 & -39.6 & -26.58 & -38.78 \\
\hline 8 & -20.04950 & -20.04958 & 20.049504 & 194.18884 & 17.2 & -2.31 & 18.00 \\
\hline 9 & -20.04950 & -20.04956 & 20.049504 & 214.22791 & -11.5 & -11.14 & -10.74 \\
\hline 10 & -20.04950 & -20.04953 & 20.049504 & 234.27795 & -12.2 & -8.39 & -11.55 \\
\hline 11 & -20.04950 & -20.04956 & 20.049504 & 254.32800 & -9.4 & -9.31 & -8.67 \\
\hline 12 & -20.04950 & -20.04953 & 20.049504 & 274.38904 & 13.7 & 9.20 & 14.35 \\
\hline 13 & -20.04950 & -20.04953 & 20.049504 & 294.42810 & -18.6 & 3.89 & -17.94 \\
\hline 14 & -20.04950 & -20.04953 & 20.049504 & 314.48364 & -18.4 & 25.37 & -17.69 \\
\hline 15 & -20.04950 & -20.04956 & 20.049504 & 334.52800 & -8.4 & -3.21 & -7.63 \\
\hline 16 & -20.04950 & -20.04956 & 20.049504 & 354.58374 & 5.0 & 5.82 & 5.80 \\
\hline 17 & -20.04950 & -20.04958 & 20.049504 & 14.61731 & -44.2 & -2.35 & -43.40 \\
\hline 18 & -20.04950 & -20.04958 & 20.049504 & 34.67834 & 18.7 & -23.76 & 19.51 \\
\hline
\end{tabular}

Tablel shows that the maximal positive position deviation of driven component is $27.02^{\prime \prime}$, while the maximal negative position deviation is $-43.04 "$. The average of position deviation is $-6.88^{\prime \prime}$. The root mean square value of position deviation is 21.01". The standard deviation of position deviation is 20.42". The root mean square value of position deviation is less than 25", which has satisfied the design requirement of antenna-driven component.

\section{Summary}

A method of accuracy measurement on rotation angle of antenna-driven component is proposed and the corresponding measurement equipment is developed. The working principles of accuracy measurement and error analysis are presented at detail. The PC application software associating with the measurement equipment is developed. The related experiments are conducted. The experiment results demonstrate the correctness and effectiveness of the method.

\section{References}

[1] Zhang Wei-Wen. Modeling and precision analysis of space-driven component. Harbin Institute of Technology University. 2009

[2] Huang Yin-guo, Lin Yu-chi, Wang Wei, Zhao Mei-rong. Laser Autocollimation Measurement of Small Angle Based on Cross Correlation. Nanotechnology and Precision Engineering. 2010.3, $8(2): 20-25$.

[3] Tao Jing-qiao, Sun Xiao-song, Li Ming. Accuracy analysis for spin satellite test turntable. Aerospace Control and Application. 2010.4, 36(2):20-24.

[4] Deng Huiyu, Wang Xinli and Ma Peisuna, A study of wavelet analysis based error 
compensation for the angular measuring system of high-precision test turntables, ISA Transactions .44,15-21(2005) 